\title{
Tratamento da leishmaniose, limitações da terapêutica atual e a necessidade de novas alternativas: Uma revisão narrativa
}

\author{
Leishmaniasis treatment, current therapy limitations and new alternative requirements: A
} narrative review

Tratamiento de leishmaniasis, limitaciones de la terapéutica actual y la necesidad de nuevas alternativas: Una revisión narrativa

Recebido: 26/05/2021 | Revisado: 04/06/2021 | Aceito: 08/06/2021 | Publicado: 22/06/2021

\author{
Alexandre Silva Santiago \\ ORCID https://orcid.org/0000-0002-7335-960X \\ Universidade do Estado da Bahia, Brasil \\ E-mail: alesantiago@live.com \\ Samuel Silva da Rocha Pita \\ ORCID: https://orcid.org/0000-0003-4053-8721 \\ Universidade Federal da Bahia, Brasil \\ E-mail samuelrpita@gmail.com \\ Elisalva Teixeira Guimarães \\ ORCID: https://orcid.org/0000-0001-9274-2957 \\ Universidade do Estado da Bahia, Brasil \\ E-mail etguimaraes@uneb.br
}

\begin{abstract}
Resumo
As leishmanioses são um grupo de doenças negligenciadas causadas por protozoários intracelulares do gênero Leishmania. Suas principais formas clínicas são a tegumentar (LT) e a visceral (LV). Atualmente a terapêutica contra a leishmaniose baseia-se na utilização de cinco fármacos: os antimoniais pentavalentes, a anfotericina B e a sua formulação lipossômica, a miltefosina, a paromomicina e a pentamidina. Estes compostos apresentam limitações que dificultam a adesão do paciente ao tratamento como: a elevada toxicidade e a necessidade de administração prolongada por via parenteral, além da possível seleção de cepas resistentes. Assim, utilizando a revisão narrativa de literatura, buscou-se elucidar um panorama do tratamento atual da leishmaniose, seus mecanismos de ação elucidados, sua toxicidade atribuída, seus efeitos adversos e vias de administração. Para tanto, os dados mais atualizados disponíveis na literatura foram trazidos para facilitar o acesso às informações sobre as opções terapêuticas, além das novas alternativas terapêuticas e perspectivas de vacinas contra esta doença negligenciada.

Palavras-chave: Doenças negligenciadas; Leishmania; Tratamento farmacológico; Mecanismos moleculares de ação farmacológica.
\end{abstract}

\begin{abstract}
Leishmaniasis groups some neglected diseases caused by intracellular protozoa Leishmania. Its main clinical forms are the tegumentary (LT) and Visceral (LV). Currently, the therapy against leishmaniasis is based on the use of five drugs: the pentavalent antimonials, amphotericin B and its liposomal formulation, miltefosine, paromomycin and pentamidine. These compounds present limitations that difficult patient's adherence to treatment, such as high toxicity and the need for prolonged parenteral administration, in addition to the selection of resistant strains. Thus, employing the literature narrative review, we brought a panorama of the current leishmaniasis treatment, its elucidated action mechanisms, attributed toxicity, adverse effects and administration routes. Aiming to point this, the more updated data available in the literature were brought to facilitate access information on therapeutic options, in addition to new therapeutic alternatives and vaccine perspectives against this neglected disease.
\end{abstract}

Keywords: Neglected diseases; Leishmania; Pharmacological treatment; Molecular mechanisms of pharmacological action.

\section{Resumen}

Leishmaniasis es un grupo de enfermedades desatendidas causadas por protozoarios intracelulares del género Leishmania. Sus principales formas clínicas son tegumentario (LT) y visceral (LV). Actualmente, la terapéutica contra leishmaniosis es basada en la utilización de cinco fármacos: los antimoniales pentavalentes, la anfotericina $\mathrm{B}$ y su formulación liposomal, la miltefosina, la paromomicina y la pentamidina. Estos compuestos presentan limitaciones que dificultan la adherencia del paciente al tratamiento como: la elevada toxicidad y la necesidad de administración prolongada por vía parenteral, así como una posible selección de cepas resistentes. Así, utilizando la revisión narrativa 
de literatura, se busca aclarar un panorama del tratamiento actual de la leishmaniasis, sus mecanismos de acción elucidados, su toxicidad, sus efectos adversos y vías de administración. Para tanto, se traen los datos más actuales disponibles en la literatura para facilitar el acceso a las informaciones sobre las opciones terapéuticas, y también de las nuevas alternativas terapéuticas y perspectivas de vacunas contra esta enfermedad desatendida.

Palabras clave: Enfermedades desatendidas; Leishmania; Tratamiento farmacológico; Mecanismos moleculares de acción farmacológica.

\section{Introdução}

As leishmanioses constituem um grupo de doenças negligenciadas, causadas por protozoários intracelulares do gênero Leishmania (Kinetoplastida, Trypanosomatidae), que se apresentam sob duas formas principais: a amastigota e a promastigota (Vasconcelos, et al., 2018). A infecção tem início quando a forma infectante (promastigota) entra em contato com o tecido do hospedeiro durante o repasto sanguíneo do mosquito flebotomíneo fêmea infectado (Ueno \& Wilson, 2012). A apresentação clínica da doença é dependente da espécie do parasito envolvido na infecção, sendo as principais formas clínicas a tegumentar (LT) e a visceral (LV), considerada mais grave devido ao seu quadro clínico, que pode levar o paciente ao óbito. A LT pode ser subdividida em: cutânea (LC), a mucosa (LM), cutânea difusa (LCD) e sua forma disseminada (LD) (Kevric, et al., 2015; Vasconcelos, et al., 2018).

Dados da Organização Pan-americana de Saúde informam que no continente Americano entre 2001-2018 foram registrados 63.331 novos casos de leishmaniose visceral e 989.096 casos de leishmaniose tegumentar (cutânea e mucosa). Do total de casos de 2018, $84 \%$ de leishmaniose tegumentar e 97\% leishmaniose visceral foram registrados pelo Brasil. É importante salientar que pacientes com LV podem apresentar hepatoesplenomegalia devido a proliferação progressiva de parasitos em macrófagos no fígado, baço e medula óssea, podendo chegar ao quadro de supressão medular. Caso não sejam tratados, poderão desenvolver pancitopenia e imunossupressão, sendo propensos a infecções concomitantes por outros microrganismos (Mcgwire \& Satoskar, 2014). No Brasil, uma das formas mais graves da leishmaniose tegumentar é a LCD, que devido a imunossupressão celular específica induzida pelo parasito, apresenta um difícil controle da infecção pelo sistema imunológico do hospedeiro, resultando no caráter evolutivo crônico e tratamento dificultado (Silveira, 2009).

Atualmente, a terapêutica contra a leishmaniose baseia-se na utilização de cinco fármacos: os antimoniais pentavalentes, a anfotericina B e a sua formulação lipossômica, a miltefosina, a paromomicina e a pentamidina. Com exceção dos compostos antimoniais, todos os outros medicamentos não foram desenvolvidos incialmente com ação leishmanicida. Além disso estão associados a deficiências graves como toxicidade, administração prolongada e possível surgimento de resistência por parte do parasito (Tiwari, et al., 2018). Tendo em vista a gravidade da doença e os problemas apresentados pela terapêutica atual, esta revisão tem como objetivo elucidar um panorama sobre o tratamento atual da leishmaniose, seus mecanismos de ação elucidados, toxicidade atribuída, efeitos adversos e vias de administração; compilando dados atualizados disponíveis em literatura para facilitar o acesso às informações sobre as opções terapêuticas, além das novas alternativas terapêuticas e perspectivas de vacinas contra esta doença negligenciada.

\section{Metodologia}

Este artigo trata-se de uma revisão narrativa de literatura, sendo assim, constituída por uma análise ampla da literatura, apropriada para descrever e discutir o desenvolvimento ou o "estado da arte" de um determinado assunto, sem estabelecer uma metodologia rigorosa e replicável em nível de reprodução de dados (Rother, 2007; Vosgerau \& Romanowski, 2014). 


\section{Resultados e Discussão}

\subsection{Terapia Atual}

A terapia medicamentosa para todas as formas de leishmaniose visa a garantia da adesão ao tratamento, o alívio dos sintomas e do desconforto causado pela doença, a administração com segurança dos medicamentos indicados, o controle e/ou a minimização da ocorrência de efeitos adversos (Brasil, 2018). No entanto, todos os fármacos disponíveis atualmente para o tratamento desta enfermidade mostram-se insatisfatórios em termos de eficácia, custo, facilidade de administração e/ou segurança (Mcgwire \& Satoskar, 2014; Murray, 2010; Hendrickx, et al., 2019).

Além disso, os antimoniais, por exemplo, aliado à longa duração do tratamento através de injeções, podem apresentar o inconveniente da dor local, por conta da via de administração intramuscular (IM), necessitando assim da alternância dos locais de aplicação, preferindo-se a região glútea. A anfotericina B, pertencente à classe IV do Sistema de Classificação Biofarmacêutica (SCB), não apresenta boas propriedades físico-químicas. Sua solubilidade em água é limitada, apresenta baixa permeabilidade em membranas, elevado peso molecular e presença de muitos grupos aceptores e doadores de ligação de hidrogênio (Barratt \& Bretagne, 2007; Fangueiro, et al., 2012). Por isso, a anfotericina B é fracamente absorvida e possui baixa biodisponibilidade oral, sendo obrigatoriamente administrada por via intravenosa (IV), demandando hospitalização do paciente, o que limita o seu uso em países pobres e em desenvolvimento (Croft \& Coombs, 2003; Bastos, et al., 2016).

A miltefosina, único fármaco de administração por via oral, pode ocasionar distúrbios gastrointestinais e toxicidade renal que levam o paciente à interrupção prematura do tratamento, o que corrobora para o aumento dos casos de resistência do parasito (Guerin, et al., 2002; Sundar, et al., 2005). Quanto à paromomicina, a necessidade de administração parenteral representa uma dificuldade para a sua adoção em países pobres e em desenvolvimento (Bastos, et al., 2016). A pentamidina apresenta como um dos efeitos secundários irreversíveis, o diabetes mellitus insulinodependente. Além disso, casos de resistência à pentamidina também têm sido descritos em literatura, como pode ser observado em mais detalhes na tabela 1 (Sundar, et al., 2006; Bray, et al., 2013).

\subsection{Os Antimoniais Pentavalentes}

Os antimoniais trivalentes $\left(\mathrm{Sb}^{\mathrm{III}}\right)$ já eram usados no Brasil em 1912 como o primeiro tratamento eficaz para leishmaniose (Vianna, 1912; Hendrickx, et al., 2019), sendo posteriormente substituídos pelos pentavalentes $\left(\mathrm{Sb}^{\mathrm{V}}\right)$ na tentativa de reduzir seus diversos efeitos secundários graves. Os antimoniais pentavalentes são os medicamentos de primeira escolha para todas as formas de leishmaniose (Kato, et al., 2014), os quais são administrados por via intramuscular (IM) ou intravenosa (IV) (Kip, et al., 2018). O antimônio pentavalente $\left(\mathrm{Sb}^{\mathrm{V}}\right)$ é considerado um pró-fármaco, que é convertido na forma ativa trivalente ( $\mathrm{Sb}^{\mathrm{III}}$ ) sob condições de pH baixo nos fagócitos hospedeiros (Singh, et al., 2012). Este mecanismo de ação dentro do organismo gera toxicidade tanto contra o hospedeiro quanto contra o parasito (Kato, et al., 2014). Atualmente, dois complexos

de antimoniais pentavalentes estão disponíveis comercialmente: o antimoniato de meglumina (Glucantime®) e o estibogluconato de sódio (Pentostan ${ }^{\circledR}$ ) (Tiwari, et al., 2018). No Brasil, o medicamento de escolha é o Glucantime ${ }^{\circledR}$ (Sereno, et al., 2000).

$\mathrm{O} \mathrm{Sb}^{\mathrm{III}}$ induz apoptose nos parasitos através da inibição da tripanotiona redutase (TR) (Tiwari, et al., 2018). A TR é uma enzima essencial e exclusiva dos parasitos da família Trypanosomatidae, responsável pelo metabolismo redox do tiol, sendo assim essencial para a manutenção da vida nos protozoários do gênero Leishmania (Beig, et al., 2015). A TR é uma flavoenzima dependente de NADPH capaz de catalisar a redução do dissulfeto de tripanotiona [TS2] em ditiol tripanotiona T(SH)2] (Flohé, 2011; Krauth-Siegel, et al., 2005). Desse modo, através da TR a tripanotiona é mantida reduzida (KrauthSiegel \& Comini, 2008). Sendo assim, esta enzima age como um antioxidante parasitário de defesa ao estresse oxidativo do hospedeiro (Dukhyl, 2019). 
$\mathrm{O} \mathrm{Sb}^{\mathrm{v}}$ também apresenta atividade antiparasitária através da inibição da atividade da topoisomerase (No, 2016). As topoisomerases são um grupo de enzimas responsáveis por controlar a homeostase topológica do DNA durante os processos de replicação, transcrição e condensação da cromatina; estando envolvidas diversos processos celulares, incluindo ciclo celular, proliferação e diferenciação celular (Sun, et al., 2020). Na leishmania, a enzima se encontra presente no núcleo e na única mitocôndria existente. Existem diferenças claras na estrutura e expressão entre as enzimas do parasito e do hospedeiro, demonstrando assim o grande potencial terapêutico dos inibidores topoisomerase como droga com atividade leishmanicida (Reguera, et al., 2019). Também já foi proposto como mecanismo de ação provável a inibição da adenosina trifosfato (ATP) e da guanosina trifosfato (GTP) através da inibição do ciclo do ácido cítrico e da glicólise (Koff \& Rosen, 1994).

O uso dos antimoniais pode ocasionar dor abdominal, vômito, diarréia, náusea, dor de cabeça, fadiga, febre, tosse, erupção cutânea, pancreatite, pneumonia, insuficiência hepática, nefrotoxicidade e cardiotoxicidade. Além de todos esses inconvenientes, também é necessária a internação hospitalar para a sua aplicação por via intramuscular ou intravenosa (Hendrickx, et al., 2019).

\subsection{Anfotericina B}

A anfotericina B $(\mathrm{AmpB})$ é um metabólito antifúngico de polieno, pouco solúvel em água e possui alta afinidade por membranas contendo esterol. O ergosterol é o principal esteroide presente nas membranas plasmáticas dos parasitos do gênero Leishmania, a anfotericina liga-se ao ergosterol, causando desorganização da membrana, formação de poros aquosos, alteração da permeabilidade de membrana com extravasamento de líquidos, desequilíbrio iônico e morte do parasito (Sampaio, 2003; Kip, et al., 2018; Tiwari, et al., 2018). Sua aplicação é intravenosa, o que resulta em menor adesão do paciente ao tratamento (Hendrickx, et al., 2019). A AmpB também tem a capacidade de ligar-se ao colesterol das membranas humanas, mecanismos esse responsável por uma grande fração de seus efeitos tóxicos (Laniado-Laborín \& Cabrales-Vargas, 2009).

Dentre os efeitos adversos causados pelo uso da AmpB destacam-se a febre alta, calafrios e insuficiência renal (Freitas-Junior, 2012), além de hipocalemia e nefrotoxicidade com limitação de dose o que dificulta seu uso na clínica (Kip, et al., 2018). A nefrotoxicidade é descrita como o principal efeito adverso associado ao uso da AmpB (Hendrickx, et al., 2019). Os efeitos adversos da AmpB foram contornados por sua formulação lipossomal (AmBisome), que também foi altamente eficaz na cura de todas as formas de infecções por leishmania (Kip, et al., 2018; Tiwari, et al., 2018). Essa formulação consiste em incorporar a AmpB numa bicamada de lipossomas, o que resulta numa redução significativa da toxicidade renal relacionada à infusão. Algumas leves reações, nefrotoxicidade transitória e trombocitopenia podem ser observadas após o seu uso. Um grande inconveniente dessa formulação é o alto custo que impossibilita seu uso por populações de baixa renda, que ironicamente são os mais afetados por esse grupo de doenças (Nagle, 2014).

\subsection{Miltefosina}

A miltefosina (hexadecilfosfocolina), um análogo alquilfosfocolina, originalmente desenvolvido como agente antitumoral, atualmente é o único medicamento de uso oral disponível para o tratamento da leishmaniose (Tiwari, et al., 2018). A sua atividade antitumoral tem como alvo as vias de sinalização do fosfoinositol 3-cinase-Akt/PKB para inibir o crescimento metastático das células. Em relação a sua atividade antiparasitária, nenhum modo de ação foi ainda elucidado, porém surgiram várias hipóteses, como a indução de apoptose, distúrbio das vias de sinalização celular dependente de lipídios, alteração da composição da membrana e efeitos imunomoduladores (Dorlo, et al., 2012; Paris, et al., 2014). Além disso, a miltefosina tem a capacidade de despolarizar o potencial da membrana mitocondrial e inibir a citocromo-c oxidase, o que pode estar relacionado a morte dos parasitos por apoptose (Marinho, et al., 2011). 
A miltefosina também produz efeitos colaterais graves (Singh, et al., 2014). São relatadas nefrotoxicidade, hepatotoxicidade e teratogenicidade gastrointestinal grave, o que dificulta o seu emprego em mulheres grávidas e crianças (Dorlo, et al., 2012; Hendrickx, et al., 2019). Aliado aos efeitos adversos, sua aplicação está comprometida devido ao crescente aumento das taxas de falha terapêutica e ao lento, porém inevitável, surgimento de resistência do parasito ao fármaco (Cojean, et al., 2012; Rijal, et al., 2013; Hendrickx, et al., 2019).

\subsection{Paromomicina}

A paromomicina, um antibiótico aminoglicosídico, possui atividade antileishmania e antibacteriana, no entanto, este medicamento não é uma boa opção para o tratamento de pacientes com leishmaniose por conta de sua baixa absorção e administração através de injeções intramusculares (Tiwari, et al., 2018; Hendrickx, et al., 2019). Estudos informam que o mecanismo de ação da paromomicina envolve a inibição da síntese de proteínas do protozoário ligando-se à subunidade ribossômica 30S, resultando no acúmulo de complexos ribossômicos anormais 30S-50S e, finalmente, causando a morte celular (Kip, et al., 2018). Outros estudos relacionam a inibição da síntese de proteínas e a alteração no potencial da membrana mitocondrial com a morte parasitária (Chawla, et al., 2011). A paromomicina apresenta como efeitos adversos mais comuns a nefrotoxicidade, a ototoxicidade e a hepatotoxicidade (Hendrickx, et al., 2019).

\subsection{Pentamidina}

A pentamidina é um derivado sintético da amidina usado como terapia de segunda linha contra a leishmaniose, uso este limitado devido à baixa eficácia e a sua administração por via IM ou, preferencialmente, por administração IV (Kip, et al., 2018; Hendrickx, et al., 2019). O mecanismo de ação da pentamidina não é claro, mas a mitocôndria foi considerada um importante alvo (Coelho, et al., 2007). Sugere-se que o composto penetra nos parasitos através de transportadores de arginina e poliamina e iniba a topoisomerase mitocondrial II (Singh, et al., 2016). Estudos bioquímicos indicaram que clones de promastigotas de Leishmania donavani e Leishmania amazonensis apresentam resistência ao medicamento devido à diminuição da captação, seguido por aumento do efluxo de medicamentos (Singh, et al., 2012). Seus efeitos adversos incluem a indução de diabetes mellitus insulino-dependente, hipotensão, efeitos gastrointestinais, hipoglicemia grave, choque, miocardite, cardiotoxicidade e toxicidade renal (Kip, et al., 2018; Hendrickx, et al., 2019). 
Tabela 1: Principais drogas utilizadas na clínica para tratamento das Leishmanioses.

\begin{tabular}{|c|c|c|c|c|}
\hline Fármacos & Mecanismos de ação & $\begin{array}{c}\text { Via de } \\
\text { Administração }\end{array}$ & Efeitos Adversos & Referências \\
\hline $\begin{array}{c}\text { Antimoniais } \\
\text { pentavalentes }\end{array}$ & $\begin{array}{c}\text { Redução do } \mathrm{Sb+5} \\
\text { a Sb+3 dentro do organismo; } \\
\text { Apoptose, através da inibição } \\
\text { da tripanotiona redutase; } \\
\text { Inibição da atividade da } \\
\text { topoisomerase. }\end{array}$ & IM, IV & $\begin{array}{c}\text { Dor abdominal, vômito, } \\
\text { diarreia, náusea, dor de cabeça, } \\
\text { fadiga, febre, tosse, erupção } \\
\text { cutânea, pancreatite, } \\
\text { pneumonia, insuficiência } \\
\text { hepática, nefrotoxicidade e, } \\
\text { cardiotoxicidade. }\end{array}$ & $7,10,20,29$ \\
\hline Miltefosina & $\begin{array}{c}\text { Inibição do citocromo-c } \\
\text { oxidase através da } \\
\text { despolarização do potencial da } \\
\text { membrana mitocondrial, o que } \\
\text { pode estar relacionado à morte } \\
\text { de parasitos por apoptose. }\end{array}$ & VO & $\begin{array}{l}\text { Toxicidade gastrointestinal, } \\
\text { nefrotoxicidade, } \\
\text { hepatotoxicidade e } \\
\text { teratogenicidade. }\end{array}$ & $7,10,37,39$ \\
\hline Paromomicina & $\begin{array}{c}\text { Inibição da síntese de } \\
\text { proteínas do protozoário } \\
\text { ligando-se à subunidade } \\
\text { ribossômica } 30 \mathrm{~S} \text {, resultando } \\
\text { no acúmulo de complexos } \\
\text { ribossômicos anormais } 30 \mathrm{~S}- \\
\text { 50S, causando a morte celular. }\end{array}$ & IM ou TÓPICO & $\begin{array}{l}\text { Nefrotoxicidade, ototoxicidade, } \\
\text { hepatotoxicidade. }\end{array}$ & $7,10,21$ \\
\hline Pentamidina & $\begin{array}{c}\text { Inibição o sistema de } \\
\text { transporte ativo; Inibição da } \\
\text { topoisomerase mitocondrial II. }\end{array}$ & $\begin{array}{l}\text { IM ou, } \\
\text { preferencialmente } \\
\text { IV }\end{array}$ & $\begin{array}{l}\text { Cardiotoxicidade, hipotensão e } \\
\text { efeitos gastrointestinais, bem } \\
\text { como, indução de diabetes } \\
\text { mellitus insulino-dependente. }\end{array}$ & $7,10,22,45$ \\
\hline
\end{tabular}

Fonte: Autores.

\subsection{Novas Alternativas Terapêuticas}

Apesar do recente desenvolvimento de vacina para cães, até o momento, não há uma vacina para humanos, tornando o tratamento farmacológico a única opção, já que o controle do vetor não tem se mostrado uma efetiva medida de prevenção da leishmaniose (Luna \& Campos, 2020; Bastos, et al, 2006). Nos últimos anos, no Brasil, foram licenciadas duas vacinas para administração nos cães (Martin, et al., 2014; Reguera, et al., 2016; Beugnet, et al., 2018; Cotrina, et al., 2018).

A Leishmune® (Zoetis, Brasil) é uma vacina de segunda geração e foi licenciada no Brasil em 2003 para proteger os cães contra L. donovani (Reguera, et al., 2016; Lopes, et al., 2018). Os cães vacinados não apresentam sinais clínicos nem parasitos em amostras de pele, linfonodos e sangue (Nogueira, et al., 2005; Vulpiani, et al., 2011), o que contribui para a redução da prevalência e incidência da doença nas populações, tanto de cães quanto de seres humanos em zonas endêmicas, diminuindo o risco de transmissão da doença para os flebótomos (Palatnik-De-Sousa, et al., 2009; Otranto \& Dantas-Torres, 2013; Reguera, et al., 2016).

A Leish-Tec® (Hertape Calier Saúde Animal SA, Brasil) foi aprovada em 2013, sendo a primeira vacina recombinante aprovada no Brasil (Reguera, et al., 2016; Coura-Vital, et al., 2018). O estudo comparativo desenvolvido entre animais vacinados com Leishmune $®$ e Leish-Tec®, demonstrou que não há diferença significativa na carga parasitária presente nos animais vacinados com ambas as vacinas, bem como baixas taxas de transmissão dos cães para os flebótomos em testes de xenodiagnóstico (Fernandes, et al., 2014). O que só reforça a capacidade de redução da prevalência e incidência da doença nas populações, tanto de cães quanto de seres humanos. 
Mesmo com esse grande avanço na área veterinária, ainda há um longo caminho até que se produza uma vacina de uso humano contra a leishmaniose, tendo em vista que esta deve ser segura, eficaz, estável e de baixo custo. Além disso, a maior incidência da doença acontece em países em desenvolvimento, sendo, portanto, um grande desafio uma vez que são necessários grandes investimentos financeiros, de infraestrutura e em pesquisa para que esse objetivo possa ser alcançado (Silva, et al., 2013).

Apesar de nenhuma vacina humana ter atingindo o status de registro, duas vacinas humanas foram testadas em ensaios clínicos. Uma vacina de primeira geração de leishmania autoclavada foi testada em um estudo de fase II com crianças no Sudão e mostrou-se imunogênica, segura e teve conversão positiva do teste cutâneo de leishmania. Sua segurança e a imunogenicidade foram observadas em $76 \%$ dos voluntários humanos que produziram IFN- $\gamma$ em resposta ao lisado do parasito (Luna \& Campos, 2020; Palatnik-De-Sousa \& Nico, 2020).

A segunda vacina humana testada em ensaios clínicos foi a LeishF3, que é uma vacina multicomponente e está em fase II. A vacina demostrou uma robusta resposta imune contra leishmaniose visceral durante o ensaio de fase I com humanos sadios nos Estados Unidos. Além de ter sido considerada segura, também foi detectada secreção aumentada de citocinas IFN- $\gamma$, TNF- $\alpha$, IL-2, IL-5 e IL-10. Em um estudo realizado em Bangladesh foi encontrada uma forte resposta de citocinas para cada componente da vacina em pacientes com leishmaniose visceral (Moafi, et al., 2019; Luna \& Campos, 2020; Palatnik-De-Sousa $\&$ Nico, 2020).

É importante ressaltar que a ampla utilização da monoterapia, levou ao surgimento de cepas resistentes aos fármacos utilizados. Dessa forma, as combinações mostraram-se boas alternativas e têm apresentado diversas vantagens em relação à monoterapia, incluindo a minimização dos efeitos tóxicos através da redução do tempo de tratamento; maior adesão ao tratamento; redução dos custos diretos e indiretos para o sistema de saúde e a atenuação do desenvolvimento de resistência por parte do parasito (Alvar, et al, 2006; Bastos, et al, 2016). Dentre estas combinações terapêuticas estão: estibogluconato de sódio e paromomicina; antimoniato de meglumina e paromomicina; anfotericina B e miltefosina; anfotericina B e paromomicina e, por fim, miltefosina e paromomicina (Alvar, et al, 2006; Sundar \& Olliaro, 2007; Bastos, et al, 2016).

Em 2019 a DNDi finalizou um ensaio de fase II e iniciou um de fase III, combinando termoterapia e miltefosina para o tratamento da leishmaniose cutânea (LC) não complicada. Nesse estudo foi avaliada a eficácia e a segurança da combinação de termoterapia (uma sessão) + miltefosina (dose padrão de $2,5 \mathrm{mg} / \mathrm{kg} / \mathrm{dia}$ por 21 dias, via oral), em comparação à apenas uma sessão de termoterapia no tratamento da LC não complicada no Peru e na Colômbia. Esse estudo contou com 130 participantes e os resultados preliminares na análise por intenção de tratar (ITT) mostraram evidências a favor da combinação. $O$ estudo de fase III tem como objetivo determinar se a combinação de tratamentos não é inferior ao tratamento padrão de primeira linha (antimoniato de meglumina de $20 \mathrm{mg} / \mathrm{kg} /$ dia por 20 dias, uso parenteral) e a monoterapia com miltefosina (dose padrão de 2,5 $\mathrm{mg} / \mathrm{kg} /$ dia por 28 dias, por via oral) (Boni, 2019). Ainda em 2019 a DNDi desenvolveu um diverso portfólio, totalizando 21 projetos com dez novas classes químicas (quatro séries líderes, quatro candidatos pré-clínicos e dois candidatos clínicos) com diferentes mecanismos de ação contra os parasitos do gênero Leishmania. Esta cadeia de desenvolvimento oferece uma base sólida para que se avance com uma ou mais novas terapias orais seguras e acessíveis para a leishmaniose visceral e cutânea (DNDi, 2019).

Além da termoterapia anteriormente citada, outras terapias alternativas também podem ser aplicadas a leishmaniose, a exemplo da terapia fotodinâmica, que surgiu como uma alternativa de tratamento promissora, que permite a administração local com menos efeitos colaterais (Pinto, et al., 2016). Esse tipo de terapia é baseada na aplicação de uma substância fotossensível na lesão-alvo, que, ao receber luz de comprimento de onda específico, é ativada desencadeando uma série de reações na presença de oxigênio molecular, levando a produção de espécies reativas de oxigênio, que resulta na morte celular e destruição do tecido doente (Machado, 2000; Bacelar, et al., 2015). Os fotossensibilizadores podem ser corantes, que são 
substâncias fotossensíveis, geralmente não tóxicas para aplicação tópica, caracterizadas por uma alta capacidade de absorver a luz visível (Volpe, et al., 2018). Um exemplo de corante fotossensibilizador é o Rosa Bengala, derivado do grupo xanteno; o qual seus seus derivados éster metílico de rosa bengala (RBMET) e éster butílico (RBBUT) mostraram-se eficazes contra $L$. amazonensis no tratamento da leishmaniose tegumentar (Navasconi, et al., 2017). Em 2018, um estudo utilizando laser de dióxido de carbono (CO2) fracionado seguido de aplicação tópica de estibogluconato de sódio demostrou que este é um tratamento seguro e promissor para infecção por leishmaniose cutânea em crianças. Dez crianças foram tratadas, destas, apenas uma não respondeu ao tratamento, as outras nove obtiveram cura clínica, com alta tolerância ao tratamento e nenhum efeito adverso observado ou relatado (Hilerowicz, et al., 2018). Outros exemplos de terapias alternativas são a crioterapia e eletroterapia (Zulfiqar, et al., 2017).

A Química Medicinal é um ramo da ciência que versa sobre a descoberta e o desenvolvimento de fármacos (Taylor \& Triggle, 2006). Através dela é possível: a identificação e interpretação do mecanismo molecular de ação de compostos biologicamente ativos; a investigação das relações entre a estrutura química e a atividade biológica, o que contribui significativamente para o melhor entendimento dos processos fisiopatológicos, bem como para o desenvolvimento de novos medicamentos mais seguros e eficazes (Amaral, et al., 2017); além destes, o melhoramento das propriedades medicamentosas, por meio de modificações específicas nas estruturas químicas, viabilizando-se a síntese de novos e mais potentes compostos com características farmacofóricas comuns a compostos antileishmania (Rashid, et al., 2016).

Uma vertente recente da Química Medicinal são os métodos in silico (computacionais). Como exemplo temos a Triagem Virtual, que surgiu na identificação de novos candidatos a fármacos como alternativa a triagem de alto rendimento (HTS). Sua principal vantagem é que bibliotecas virtuais (quimiotecas) contendo diversos compostos (> 500) são avaliadas através de diferentes ferramentas computacionais, resultando num pequeno conjunto de compostos priorizados para ensaios secundários. Economiza-se assim insumos, matérias-primas e reagentes. Além disso esses métodos in silico possibilitam também a identificação de: propriedades moleculares (drug-like e estruturais), similaridade 2D, a presença de grupos farmacofóricos (Piccirillo \& Amaral, 2018), cardiotoxicidade, capacidade de agregação, presença de falsos positivos (PAINS) e a toxicidade de moléculas. Além disso, os estudos de Triagem Virtual podem ser aplicados a compostos de origem sintética quanto natural. O Banco de Dados de Produtos Naturais do Semi-Árido Baiano (NatProDB, www.natprodb.uefs.br), por exemplo é uma base de dados com grande diversidade química o que pode favorecer a descoberta de novas entidades químicas (Paixão \& Pita, 2019).

Adicionalmente, outra estratégia que tem sido empregada para melhorar a eficiência desse tratamento é a busca de novas formas farmacêuticas sítio-específicas. Os sistemas mais utilizados são os lipossomas e as nanopartículas; e seus principais benefícios são: a redução dos efeitos adversos, através direcionamento do fármaco aos tecidos ou células afetadas; a proteção do fármaco contra a degradação em fluidos biológicos, o que culminou na possibilidade de administração por novas vias; além do aumento da biodisponibilidade, possibilitando a administração por via oral (Bastos, et al, 2016).

\section{Conclusão}

Embora a Leishmaniose se apresente como um problema de saúde pública, diversos trabalhos apontam inúmeras limitações às terapêuticas atuais, incluindo toxicidade e alto custo. Além disso, exceto a miltefosina que é de administração oral, todos os outros requerem administração parenteral, evidenciando assim, a necessidade de opções terapêuticas mais eficazes, mais seguras e que apresentem vias de administração mais convenientes, a fim de facilitar a adesão ao tratamento e consecutivamente sua taxa de sucesso.

Sendo assim, o presente trabalho expõe os principais problemas das terapias atuais, as alternativas que vêm sendo empregadas e perspectivas futuras de novos tratamentos, reforçando a necessidade de busca por outras alternativas 
terapêuticas. Nesse contexto, essas novas opções terapêuticas podem ser reveladas de uma ou mais classes químicas diferentes das utilizadas atualmente, tornando notável que os estudos de novos protótipos devam ser fortemente apoiados como ferramentas de controle de riscos e agravos à população e à saúde pública.

\section{Referências}

Alvar, J., Croft, S., Olliaro, P., \& David, H. M. (2008). Chemotherapy in the treatment and control of Leishmaniasis. Advances in Parasitology. 223-274.

Amaral, A. T., Andrade, C. H., Kummerle, A., \& Guido, R. V. C. (2017). A evolução da Química Medicinal no Brasil: avanços nos 40 anos da Sociedade Brasileira de Química. Química Nova, 694-700.

Bacelar, I. O. L., Tsubone, T. M., Pavani, C., \& Baptista, M. S. (2015). Eficiência fotodinâmica: da fotoquímica molecular à morte celular. Int J Mol Sci. p. 20523-59.

Barratt, G., \& Bretagne, S. (2007). Optimizing efficacy of amphotericin B through nanomodification. The International Journal of Nanomedicine, p. 301.

Bastos, M. M., Boechat, N., Hoelz, L. V. B., \& de Oliveira, A. P. (2016). Quimioterapia Antileishmania: Uma Revisão da Literatura. Rev. Virtual Quim., 8 (6), 2072-2104.

Beig, M., Oellien, F., Garoff, L., Noack, S., Krauth-Siegel, R. L., \& Selzer, P. M. (2015). Trypanothione reductase: a target protein for a combined in vitro and in silico screening approach. PLoS Negl Trop Dis. v. 9, n. 6.

Beugnet, F., Halos, L., \& Guillot, J. (2018). Textbook of Clinical Parasitology in dogs and cats. Servet editorial - Grupo Asís Biomedia, S.L.

Boni, Marina. (2019). Leishmaniose: estudo de combinação de Fase III. DNDi América Latina. Disponível em: <https://www.dndial.org/2019/comunicacao-einformacao/leishmaniose-estudo-de-combinacao-de-fase-iii/>.

Brasil, Ministério da Saúde. (2018). Manejo Terapêutico de Pacientes com Leishmaniose Tegumentar Americana (LTA). Santa Catarina, 13 p. Disponível em <http://www.dive.sc.gov.br/conteudos/publicacoes/ManualLTAvisualiza\%C3\%A7\%C3\%A3o.pdf>.

Bray, P. G., Barrett, M. P., Ward, S. A., \& De Koning, H. P. (2013). Pentamidine uptake and resistance in pathogenic protozoa: past, present and future. Trends in Parasitology. 19, 232.

Chawla, B., Jhingran, A., Panigrahi, A., Stuart, K. D., \& Madhubala, R. (2011). Paromomycin affects translation and vesicle-mediated trafficking as revealed by proteomics of paromomycin susceptible resistant Leishmania donovani. PLoS One. 6(10).

Coelho, A. C., Messier, N., Ouellette, M., \& Cotrim, P. C. (2007). Role of the ABC transporter PRP1 (ABCC7) in pentamidine resistance in Leishmania amastigotes. Antimicrob Agents Chemother. 51, p.3030-2.

Cojean, S., Houzé, S., Haouchine, D., Huteau, F., Lariven, S., Hubert, V., \& Matheron, S. (2012). Leishmania resistance to miltefosine associated with genetic marker. Emerg Infect Dis. 18, 704-706.

Cotrina, F. J., Iniesta, V., Monroy, I., Baz, V., Hugnet, C., Marañon, F., A\& lonso, C. (2018). A large-scale field randomized trial demonstrates safety and efficacy of the vaccine LetiFend against canine leishmaniosis. Vaccine. 36, 1972-1982.

Coura-Vital, W., Leal, G. G. De A., Marques, L. A., Pinheiro, A. Da C., Carneiro, M., \& Reis, A. B. (2018). Effectiveness of deltamethrin-impregnated dog collars on the incidence of canine infection by Leishmania infantum: A large scale intervention study in an endemic area in Brazil. PLoS one. 13(12).

Croft, S. L., \& Coombs, G. H. (2003). Leishmaniasis current chemotherapy and recent advances in the search for novel drugs. Trends in Parasitology. 19, 502.

DNDi, (2019). Iniciativa Medicamentos para Doenças Negligenciadas. Rumo a uma nova geração de tratamentos para as leishmanioses. DNDi América Latina. <https://dndi.org/wp-content/uploads/2019/09/DNDi_Leishmaniasis_2019_POR.pdf>.

Dorlo, T. P., Balasegaram, M., Beijnen, J. H., \& De Vries, P. J. (2012). Miltefosine: a review of its pharmacology and therapeutic efficacy in the treatment of leishmaniasis. J. Antimicrob. Chemother. 67, 2576-2597.

Dukhyil A. A. A. B. (2019). Targeting Trypanothione Reductase of Leishmanial major to Fight Against Cutaneous Leishmaniasis. Infect Disord Drug Targets. $19,388-393$.

Fangueiro, J. F., Marques, I. R., Severino, P., Santana, M. H. A., \& Souto, E. B. (2012). Desenvolvimento, produção e caracterização de nanocristais de fármacos pouco solúveis. Química Nova. 35, 1848-1853.

Fernandes, C. B., Junior, J. T. M., Jesus, C., Souza, B. M., Larangeira, D. F., Fraga, D. B., Barrouin-Melo, S. M. (2014). Comparison of two commercial vaccines against visceral leishmaniasis in dogs from endemic areas: IgG, and subclasses, parasitism, and parasite transmission by xenodiagnosis. Vaccine. $1287-1295$.

Flohé, L. (2012). The trypanothione system and the opportunities it offers to create drugs for the neglected kinetoplast diseases. Biotechnology Advances. 30, 294-301.

Freitas-Junior, L. H., Chatelain, E., Kim, H. A., \& Siqueira-Neto J. L. (2012). Visceral leishmaniasis treatment: What do we have, what do we need and how to deliver it?. Int. J. Parasitol. Drugs Drug Resist. 2, 11-19. 
Guerin, P. J., Olliaro, P., Sundar, S., Boelaert, M., Croft, S. L., Desjeux, P., Wasunna, M. K., \& Bryceson, A. D. M. (2002). Visceral leishmaniasis: current status of control, diagnosis, and treatment, and a proposed research and development agenda. The Lancet Infectious Diseases. v. 2 , p. 494.

Hendrickx, S., Caljon, G., \& Maes, L. (2019). Need for sustainable approaches in antileishmanial drug Discovery. Parasitology Research.

Hilerowicz, Y., Koren, A., Mashiah, J., Katz, O., Sprecher, E., \& Artzi, O. (2018). Fractional ablative carbon dioxide laser followed by topical sodium stibogluconate application: A treatment option for pediatric cutaneous leishmaniasis. Pediatr Dermatol. v.35, n.3, p. 366-369.

Kato, K. C., Morais-Teixeira, E., Reis, P. G., Silva-Barcellos, N. M., Salaün, P., Campos, P. P., Corrêa-Junior, J. D., Rabello, A., Demicheli, C., \& Frézarda, F. (2014). Hepatotoxicity of Pentavalent Antimonial Drug: Possible Role of Residual Sb(III) and Protective Effect of Ascorbic Acid. Antimicrobial Agents and Chemotherapy. v.58, p.481.

Kevric, I., Cappel, M. A., \& Keeling, J. H. (2015). New World and Old World Leishmania Infections a Practical Review. Dermatologic Clinics. 33 , 579.

Kip, A. E., Schellens, J. H. M., Beijnen, J. H., \& Dorlo, T. P. C. (2018). Clinical Pharmacokinetics of Systemically Administered Antileishmanial Drugs. Clin Pharmacokinet. v.57, p.151-176.

Koff A. B., \& Rosen T. (1994). Treatment of cutaneous leishmaniasis. J Am Acad Dermatol, 31, 693-708.

Krauth-Siegel R. L., \& Comini, M. A. (2008). Redox control in trypanosomatids, parasitic protozoa with trypanothione-based thiol metabolism. Biochim Biophys Acta.

Krauth-Siegel, R. L., Bauer, H., \& Schirmer, R. H. (2005). Dithiol proteins as guardians of the intracellular redox milieu in parasites: old and new drug targets in trypanosomes and malaria-causing plasmodia. Angew Chem Int Ed Engl. 44, 690-715.

Laniado-Laborín, R., \& Cabrales-Vargas, M. N. (2009). Amphotericin B: side effects and toxicity. Rev Iberoam Micol. 26, $223-227$.

Lopes, E. G., Sevá, A. P., Ferreira, F., Nunes, C. M., Keid, L. B., Hiramoto, R. M, \& Soares, R. M. (2018). Vaccine effectiveness and use of collar impregnated with insecticide for reducing incidence of Leishmania infection in dogs in an endemic region for visceral leishmaniasis in Brazil. Epidemiology and Infection

Luna, E. J. De A., \& Campos, S. R. de S. L. da C. (2020). O desenvolvimento de vacinas contra as doenças tropicais negligenciadas. Cad. Saúde Pública. 36.

Machado, A. E. (2000). Terapia fotodinâmica: princípios, potencial de aplicação e perspectivas. Quim Nova. 23, $237-243$.

Marinho, F. D. A., Gonçalves, K. C. D. S., Oliveira, S. S. D., Oliveira, A.- C. D. S. C., Bellio, M., D'avila-Levy, C. M., Santos, A. L. S. D., \& Branquinha, M. H. (2011). Miltefosine induces programmed cell death in Leishmania amazonensis promastigotes. Mem. Inst. Oswaldo Cruz. 106, 507-509.

Martin, V., Vouldoukis, I., Moreno, J., Mcgahie, D., Gueguen, S., Cuisinier, A. (2014). The protective immune response produced in dogs after primary vaccination with the LiESP / QA-21 vaccine (CaniLeish ${ }^{\circledR}$ ) remains effective against an experimental challenge one year later. Veterinary Research. 45, 115 .

Mcgwire, B. S., \& Satoskar, A. R. (2014). Leishmaniasis: clinical syndromes and treatment. Q J Med. 107, .7-14.

Moafi, M., Rezvan, H., Sherkat, R., \& Taleban, R. (2019). Leishmania vaccines entered in clinical trials: a review of literature. Int J Prev Med. 10, 95.

Murray, H. W. (2010). Treatment of visceral leishmaniasis in 2010: direction from Bihar state, India. Future Microbiol. 5, 1301-1303.

Nagle, A. S., Khare, S., Kumar, A. B., Supek, F., Buchynskyy, A., \& Mathison, C. J. N., Molteni, V. (2014). Recent Developments in Drug Discovery for Leishmaniasis and Human African Trypanosomiasis. Chemical Reviews. 114, 11305.

Navasconi, T. R., Reis, V. N., Freitas, C. F., Pereira, P. C. S., Caetano, W., Hioka, N., Lonardoni, M. V. C., Aristides, S. M. A., \& Silveira, T. G. V. (2017). Photodynamic Therapy With Bengal Rose and Derivatives Against Leishmania amazonensis. J Lasers Med Sci. 8, 46-50.

No, J. H. (2016). Visceral leishmaniasis: Revisiting current treatments and approaches for future discoveries. Acta Trop. 155, 113-123.

Nogueira, F. S., Moreira, M. A. B., Borja-Cabrera, G. P., Santos, F. N., Menz, I., Parra, L. E, \& Luvizotto, M. C. R. (2005). Leishmune ® vaccine blocks the transmission of canine visceral leishmaniasis Absence of Leishmania parasites in blood, skin and lymph nodes of vaccinated exposed dogs. Vaccine. 23, 48054810

Organização Pan-Americana Da Saúde (OPAS/OMS). (2018). Leishmanioses: Informe Epidemiológico das Américas. Organização Pan-Americana da Saúde.

Otranto, D., Dantas-Torres, F. (2013). The prevention of canine leishmaniasis and its impact on public health. Trends in Parasitology. 29 , 339-345.

Paixão, V. G., \& Pita. S. S. R. (2019). In silico identification and evaluation of new Trypanosoma cruzi trypanothione reductase (TcTR) inhibitors obtained from natural products database of the Bahia semi-arid region (NatProDB). Computational Biology and Chemistry. 79, 36-47.

Palatnik-De-Sousa, C. B., Silva-Antunes, I., Morgado, A. A, Menz, I., Palatnik, M., \& Lavor, C. (2009). Decrease of the incidence of human and canine visceral leishmaniasis after dog vaccination with Leishmune ${ }^{\circledR}$ in Brazilian endemic areas. Vaccine. $27,3505-3512$.

Palatnik-De-Sousa, C. B., \& Nico, D. (2020). The delay in the licensing of protozoal vaccines: a compara - tive history. Front Immunol. v.11, p.204.

Paris, C., Loiseau, P. M., Bories, C., \& Bréard, J. (2004). Miltefosine induces apoptosis-like death in Leishmania donovani promastigotes. Antimicrob. Agents Chemother. v.48, n.3, p.852-859.

Piccirillo, E., \& Amaral, A. T. (2018). Busca virtual de compostos bioativos: conceitos e aplicações. Quím. Nova41, 662-677. 
Pinto, J .G., Fontana, L. C., Oliveira, M. A., Kurachi, C., Raniero, L. J., \& Ferreira-Strixino, J. (2016). In vitro evaluation of photodynamic therapy using curcumin on Leishmania major and Leishmania braziliensis. Lasers Med Sci. 31, 883-890.

Rashid, U., Sultana, R., Shaheen, N., Hassan, S.F., Yaqoob. F., Ahmad, M. J., Iftikhar, F., Sultana, N., Asghar, S., Yasinzai, M., Ansari, F. L., \& Qureshi, N. A. (2016). Structure based medicinal chemistry-driven strategy to design substituted dihydropyrimidines as potential antileishmanial agents. Eur J Med Chem. $10,230-44$

Reguera, R. M., Morán, M., Pérez-Pertejo, Y., García-Estrada, C., \& Balaña-Fouce, R. (2016). Current status on prevention and treatment of canine leishmaniasis. Veterinary Parasitology. 227, 98-114.

Reguera, R.M., Elmahallawy, E.K., García-Estrada, C., Carbajo-Andrés, R., \& Balaña-Fouce, R. (2019). DNA Topoisomerases of Leishmania Parasites, Druggable Targets for Drug Discovery. Curr Med Chem.26, 5900-5923.

Rijal, S., Ostyn, B., Uranw, S., Rai, K., Bhattarai, N. R., Dorlo, T. P. C., Beijnen, J. H., Vanaerschot, M., Decuypere, S., Dhakal, S. S., Lal Das, M., Karki, P., Singh, R., Boelaert, M., \& Dujardin, J-C. (2013). Increasing Failure of Miltefosine in the Treatment of Kala-azar in Nepal and the Potential Role of Parasite Drug Resistance, Reinfection, or Noncompliance. Clinical Infectious Diseases, 56, 1530-1538.

Rother E.T. (2007). Revisão sistemática x revisão narrativa. Acta Paul Enferm, 20(2):v-vi. <http://www.scielo.Br/scielo.php?script=sci_arttext\&pid+S0103$21002007000200001>$.

Sampaio R. N. R., Takano G. H., Malacarne A. C., Pereira T. R., \& de Magalhaes A. V. (2003). In vivo Terbinafine inefficacy on cutaneous leishmaniasis caused by Leishmania (Leishmania) amazonensis in C57BL/6 mice. Rev Soc Bras Med Trop.

Sereno, D., Holzmuller, P., \& Lemestre, J. L. (2000). Efficacy of second line drugs on antimonylresistant amastigotes of Leishmania infantum. Acta Tropica. 7425 .

Silva, K. L. O., Santos, D. P., Coelho, N. M. D., Silva, D. C., Okamoto, A. C., \& Junior Gaetti-Jardim, E. (2013). Vacinas contra Leishmaniose: uma revisão. Archives of Health Investigation. 2, 18-28.

Silveira, F. T., Lainson, R., De Castro Gomes, C. M., Laurenti, M. D., \& Corbett, C. E. P. (2009). Immunopathogenic competences of Leishmania (V.) braziliensis and $L$. (L.) amazonensis in american cutaneous leishmaniasis. Parasite Immunology, 31, 423 - 431.

Singh, N., Kumar, M., \& Singh, R.K. (2012). Leishmaniasis: Current status of available drugs and new potential drug targets. Asian Pac. J. Trop. Med., 5, 485-4897.

Singh, N., Mishra, B. B., Bajpai, S., Singh, R. K., \& Tiwari V. K. (2014). Natural product based leads to fight against leishmaniasis. Bioorg. Med. Chem. 22, $18-45$.

Singh, O. P., Singh, B., Chakravarty, J., \& Sundar, S. (2016). Current challenges in treatment options for visceral leishmaniasis in India: a public health perspective. Infect. Dis. Poverty.

Sun, C. H., Weng, S.C., Wu, J. H., Tung, S. Y., Su, L.H., Lin, M. H., \& Lee, G. A. (2020). DNA topoisomerase III $\beta$ promotes cyst generation by inducing cyst wall protein gene expression in Giardia lamblia. Open Biol. 102.

Sundar, S., \& Chatterjee, M. (2006). Visceral leishmaniasis current therapeutic modalities. The Indian Journal of Medical Research. v. 123 , p. 345.

Sundar, S., \& Murray, H. W. (2005). Availability of miltefosine for the treatment of kala-azar in India. Bulletin of the World Health Organization. 83, 394.

Sundar, S., \& Olliaro, P. L. (2007). Miltefosine in the treatment of leishmaniasis: Clinical evidence for informed clinical risk management. Therapeutics and Clinical Risk Management. 3, 733.

Taylor, J. B., \& Triggle, D. J. (2007). Comprehensive Medicinal Chemistry II, Elsevier.

Tiwari, N., Gedda, M. R., Tiwari, V. K., Singh, S. P., \& Singh, R. K. (2018). Limitations of Current Therapeutic Options, Possible Drug Targets and Scope of Natural Products in Control of Leishmaniasis. Mini-Reviews in Medicinal Chemistry. v. 18, n. 1.

Ueno, N., \& Wilson, M. E. (2012). Receptor-mediated phagocytosis of Leishmania: Implications for intracellular survival. Trends Parasitol. $28,35-44$.

Vasconcelos, J. M., Gomes, C. G., Sousa, A., Teixeira. A. B., \& Lima, J. M. (2018). Leishmaniose tegumentar americana: perfil epidemiológico, diagnóstico e tratamento. RBAC. 50, 221-7.

Vianna, G. (1912). Tratamento da leishmaniose tegumentar por injeções intravenosas de tártaro emético. An $7^{\circ}$ Congr Bras Med Cirurg. 4, 426-428.

Volpe, R. A. F. N., Navasconi, T. R., Reis V. N., Hioka, N., Becker, T. C. A., Lonardoni, M. V. C., Aristides, S. M. A., \& Silveira, T. G. V. (2018). Terapia fotodinâmica para o tratamento da leishmaniose tegumentar americana: avaliação da associação de terapias em camundongos infectados experimentalmente com Leishmania (Leishmania) amazonensis. J Lasers Med Sci. 9, 274-282.

Vosgerau, D. S. A. R. \& Romanowski, J. P. (2014). Estudos de revisão: implicações conceituais e metodológicas. Revista de Diálogo Educacional, (14)41, $165-189$.

Vulpiani, M. P., Iannetti, L., Paganico, D., Iannino, F., \& Ferri, N. (2011). Methods of control of the Leishmania infantum dog reservoir: State of the art. Veterinary Medicine.

Zulfiqar, B., Shelper, T. B., \& Avery, V. M. (2017). Leishmaniasis drug discovery: recente progress and challenges in assay development. Drug Discov Today. p.4. 